\title{
Protée
}

\section{« Différence et répétition ». Oeuvre de simulacre}

\section{Frédéric Boutin}

Volume 27, numéro 3, 1999

L'imaginaire de la fin

URI : https://id.erudit.org/iderudit/030577ar

DOI : https://doi.org/10.7202/030577ar

Aller au sommaire du numéro

Éditeur(s)

Département des arts et lettres - Université du Québec à Chicoutimi

ISSN

0300-3523 (imprimé)

1708-2307 (numérique)

Découvrir la revue

Citer cet article

Boutin, F. (1999). « Différence et répétition ». Oeuvre de simulacre. Protée 27(3), 119-124. https://doi.org/10.7202/030577ar

\section{Résumé de l'article}

Pourquoi, à la lecture de l'ouvrage Différence et Répétition de Deleuze, le lecteur sent-il que l'essentiel du propos du philosophe ne consiste pas essentiellement à promouvoir un savoir, une connaissance qui serait éminemment de l'ordre du contenu de l'oeuvre ? Pourquoi, traversant ce discours hermétique, a-t-il l'impression que le sens glisse devant lui et lui échappe et que précisément l'oeuvre entend jouer ainsi de lui (du sens et du lecteur) ? Cet article veut à la fois traquer cette éventuelle stratégie textuelle qui consiste à entraîner le lecteur dans les dédales d'une lecture pragmatique et définir la notion de simulacre que propose l'ouvrage. 


\section{«DIFFÉRENCE ET RÉPÉTITION》 GUVRE DE SIMULACRE}

FRÉDÉRIC BOUTIN

La pensée n'est rien sans quelque chose qui force à penser, qui fait violence à la pensée. Plus important que la pensée, il y a ce qui donne à penser; plus important que le philosophe, le poète.

Proust et les signes

Là [dans Finnegans Wake de Joyce et Le Livre de Mallarmé], l'identité de la chose lue se dissout réellement dans les séries divergentes définies par les mots ésotériques, comme l'identité du sujet lisant se dissout dans les cercles décentrés de la multilecture possible. Tout est devenu simulacre.

Différence et Répétition

Si Différence et Répétition est un grand livre, c'est peutêtre parce que $D$ eleuze, y élaborant une théorie du signe, y faisant la promotion del'apprentissage même, réalise en lui ce principe qu'il expose et qui participe de son contenu. C'est peut-être parcequel'œuvre, dans son écriture, dans sa structureformelle, et argumentativeaussi, incarneson propos et devient un signe dont on fait soi-même l'apprentissage. Différence et Répétition, pour paraphraser l'auteur, donneà penser une pensée sans image.

$N$ ous voudrions mettreen lumière cette assimilation du contenu par la forme et procéder, du même coup, au relevé des traces formelles et des moments de cette identification. On pourrait direquel'écrit transportesa solution de lecture, véhicule sa poétique, dans la mesure où, d'unepart, il prétend à l'œuvre d'art, au simulacre, et vise le renversement de la représentation et où, d'autre part, il est l'actualisation de cette prétention et de ce renversement.

U nequestion s'impose d'abord: par où commencer après la lecture de cette œuvre? Et par quoi ? Ces questions se font écho. Elles persistent. Aussi faut-il s'y accorder et admettre tout de suite qu'il n'y a pas de point de départ donné dans l'œuvre. II y en a une myriade possible, mais pas un qui soit réel, qui soit «vrai», qui soit le «bon», le «premier» point de départ où «ça» commence. Cette pro- position nous la lançons comme un avertissement et aimerions qu'on l'entende au pied de la lettre. $\mathrm{N}$ on seulement peut-on partir de n'importe où dans cette œuvre, de la première citation rencontrée, presque au hasard d'une lectureà nez levé, mais on doit le faire. $D$ ifférenceet Répétition est un ouvrage dont l'unitéréside dans levoisinage du dispars, unité multiple, unité résultant de la résonance des séries voisines. U nité qui est structure mugissante d'éléments hétéroclites, foisonnement d'histoires racontées simultanément. L'important dans une pareille œuvre, c'est de faire un commencement. C'est de partir pour inévitablement arriver à Rome. Car à y quêter éperdument l'origine, de nature conceptuelle, logique ou propositionnelle, à y chercher le point de départ, là où l'histoire commence, on est certain d'attendre longtemps. On est assuré d'errer dans les profondeurs de l'œuvre. Submergé. Et j'ajoute: tant qu'on cherche, tant qu'on poursuit cette origine dans letexte, avide decommencement, on entredansl'airedu texte, on participe pleinement à son jeu. N ous sommes follement entraînés, nous lecteurs, par lemouvement del'écriture. $N$ ous répondons à cette écriture, nous sommes «joués» par elle. N ous sommes dissous «dans les cercles décentrés dela multi-lecture possible». Le texte devient un signe dont nous faisons l'apprentissage, signe dynamiquequi entraînelelecteur dans le mouvement du décodage. II est le labyrinthe que hante l'écho du «monstre» parcourant ses corridors. Labyrinthe agile à traquer son marcheur. A riane s'est pendue, dit D eleuze, lasse d'attendre Thésée, étourdi, éperdu, par le labyrinthe. Lelecteur demêmesefait «étourdir » par letexte de D eleuze et le sera jusqu'à ce qu'il se décide à tirer luimême un fil, question de ne pas se diriger seul dans ce labyrinthe, question dese donner l'impression d'être guidé, de ne pas être mené n'importe où. V ers la sortie ou vers le monstre? Probablement que ni l'une ni l'autre n'est la véritable destination. Thésée erre dans les galeries. Et seul lui ne sait pas que c'est pour toujours. 
M ais peut-être pouvons-nous partir de ce lieu précis où le texteprend au piègelelecteur, vraisemblablestratégie textuelle deD ifférenceet Répétition: O N (letexte, l'auteur, lenarrateur) sait que le lecteur a besoin d'un point de départ à sa lecture. ON sait que ce point de départ, s'il ne lui est pas révélé, sera instamment cherché par le lecteur. Alors vient l'idée de ne pas donner ce point de départ, ou plutôt de n'en pas donner qu'un. Aucun réel, plusieurs possibles. D ès ce moment, $0 \mathrm{~N}$ peut être certain que le lecteur est pris dans le mouvement forcené et effréné de l'apprentissage. Le jeu est lancé.

\section{M odèle, copie, simulacre}

On serait tenté de reconnaître, au premier coup d'œil, que tout le système argumentatif de D ifférence et Répétition est mené par une instance dialectique qui a le sens de la contradiction. On serait tenté d'y reconnaître une inté ressante entreprise de déni où le refus logique d'une proposition de départ participe à la construction du propos et se retrouveau principemême desa cohérence. Letexte, diraiton, est constituéd'objets conceptuelss'opposant à un premier modèle logique, contredisant une proposition de départ qu'il s'agissait de renverser; et, de fil en aiguille, il arrive à l'élaboration d'une structure logique, d'un système cohérent en lui-même, mais dont les assises sont figurées en dehors de ce système, précisément dans le modèle qui a servi de point de départ à l'argumentation. Probablement, les choses se passent-elles souvent ainsi dansl'ouvrage qui nous intéresse. Et parce qu'il «renverse le modèle», il est tentant de voir dans le simulacre, concept des plus utilisés dans l'œuvre, et qui constituera principalement l'objet de notre présent propos, cemêmeélément de contradiction, cettecontrepartie logique dont il est ici question. N ous verrons qu'il en va tout autrement et quele simulacre, au contraire, devient un signe qui échappeà la dynamique dialectique. $Q$ u'il devient un élément qui dénie peut-être le modèle, mais à un tout «autre» niveau.

Prenons l'exemple de la représentation (tirons le fil de la représentation), considérant que le projet del'œuvre vise précisément son renversement. «C e que l'on reproche à la repré sentation c'est d'en rester à la forme d'identité, sous le double rapport de la chose vue et du sujet voyant» (D R : 94). D eleuze pose dans cette définition les trois prédicats qui fondent le procès représentatif, soit le sujet-interprétant, l'objet-interprété et l'identité, à la fois de l'objet et du sujet. Son discours consistera à renverser ces données de la représentation.

N ousferons appel aux notions platoniciennes demodèle, decopie et desimulacre, dont le philosophefait grand usage, afin de poser le problème de la représentation, tel qu'il apparaît dans I'ouvrage. O ù le modèle est le M ême, I'Identique, I'identité dans le concept; et la copie, le passage de cette identité dans un autre élément, dans un autre corps. Selon ce point devue, l'identité est un phénomèneintérieur, car c'est l'identique au cœur de la chose qui fonde la ressemblance. «[...] le modèle ne peut être défini que par une position d'identité comme essence du M ême; et la copie, par une affectation de ressemblance interne comme qualité du Semblable [...]» (D R : 341). Ainsi, le M ême, I'Identique, inaugure et fonde le monde de la représentation.

Leprojet avouédu livre concerne visiblement letroisième élément du système: il faut «[...]dénier leprimat d'un 0 riginal sur la copie, d'un modèle sur l'image. Et gl orifier le règne des simulacres et des reflets» (DR: 92). N ous voilà au vif du propos: de quelle manière le simulacre échappe-t-il au fondement, au M ême? De quelle manière renverset-il le modèleet la copie, dont, il faut bien le voir, il est issu (pas de simulacre sans modèle). Comment arrive-t-il à nier l'identité de son origine, non pas à simplement contester cette origine mais en rêvant de totalement s'en affranchir? Éternel «effondement», sel on letermemême de D eleuze. Simulacre, enfant du miracle ou de la résurrection. Sans ressemblance aucune avec aucune espèce, aucun genre? Le simulacreest le plus haut espoir de D eleuze, il est lelieu où la représentation et I'identique, fondements de la pensée occidentale, sont vaincus, dissous.

Le philosophe poursuit: «[...] II faut que la chose ne soit rien d'identique, mais soit écartelée dans une différence où s'évanouit l'identitédel'objet vu commedu sujet voyant» (DR : 79). Effondrement del'identité. D 'une part dansl'objet qui ne porte plus sa signification implicite, en filigrane dans son corps, dans son être, dansl'objet qui n'enroule plus «en lui » (D R : 35) son sens. II est tout à fait ouvert au monde, aux événements, ouvert pour recevoir de l'extérieur une signification. Et le sujet, dans le simulacre, d'autre part, n'est plus l'instance qui, se représentant l'objet, est en mesure de I'identifier, de se le remémorer et de le reconnaître. Le sujet n'est plus le dernier maillon de la chaîne interprétative, il est dissous et la sentence de la signification ne lui appartient plus. D eleuze parle d'un sujet fêlé par où se manifeste un point de vue supérieur, capable, lui, de sanctionner le sens. II rêve d'un monde où toute chose, tout sujet ou tout évé nement est «effondé». Réinventer le monde à chaque mouvement de pensée et que chaque pensée soit une agression. Aussi projette-t-il son rêve dans le simulacre, dernier espoir immoral du philosophe, où le sans-fond, l'«effon- 
dement», gronde sousla chose, sous la représentation. Cette dernière, dit l'auteur, est «étrangement coudée», dans la mesure où elle est, d'une part, appelée à être fondée par I'identique et ainsi faire partiedu monde de la représentation, mais que, d'autre part, elleest appeléeà sombrer vers ce sansfond qui l'appelle, et risque à tout moment de la renverser. D eleuze rêve d'une pensée qui a sombré dans le sans-fond, d'une penséenaufrage qui appartiendrait au monde des forces pures, des intensités, du mouvement, bref au monde du simulacre. «[...] la pensée moderne naît de la faillite de la représentation, commedela pertedesidentités, et dela découverte de toutes les forces qui agissent sous la représentation de l'identique» (D R : 1). C es forces, ces ombres agissant sous la représentation, c'est précisément au monde du simulacre qu'elles appartiennent et c'est précisément à la représentation qu'elles échappent.

\section{Simulacre et art}

[...] On sait que l'ourvre d'art moderne tend à réaliser ces conditions: elledevient en cesensvéritablethêâtre. Thếtresans rien defixeou labyrinthesansfil. L'ouvred'art quittele domaine de la représentation pour devenir «expérience». (D R : 79)

D eleuzea trouvéla nature du simulacre. Et la trouve précisément hors de la philosophie: dans l'art, la littérature, le cinéma, la peinture et le théâtre. D ès l'introduction, il compare son livre, non pas à un manuel ou à un traité d'histoire dela philosophie, ni encoreà un traitéthéoriquequelconque, mais à un «roman policier» et à un roman de «sciencefiction ». Roman policier en cela queles concepts de D ifférence et Répétition doivent intervenir dans «une zone de présence afin de résoudreunesituation locale»; science fiction en cela que l'auteur, n'oubliant pas qu'il crée tout de même une œuvre de philosophie où doit se faire entendre une parole sérieuse, ne respecte pas la rigueur scientifique requise, ni dans la construction ni dans la cohérence de son propos.

D eleuze convieensuitel'art pictural et annonceque «[...] la théorie de la pensée est comme la peinture, elle a besoin de cette révolution qui la fait passer de la représentation à l'art abstrait; tel est l'objet d'une théorie de la pensée sans image» (D R : 354). Formule éclatante, envolée idéaliste, mais qui ne révèle pas moins le lieu d'où parle $D$ eleuze, et projette vivement ses intentions: parler de la théorie sur les bases de la fiction. Faire un roman dont le reflet, l'ombre, est œuvre théorique, scientifique: simulacre accompli. Le passage de la représentation à l'art abstrait, c'est celui du fondement à I'«effondement», de la copie au simulacre, de l'I dentique à la multiplicité, du M ême à l'Autre, du concept à son actualisation, de la sédentarité au nomadisme, de la théorie à sa «fictionnalisation » dansl'exercice del'écriture. L'I dentique, dis-je, c'est l'ouvrage philosophique tel que la tradition en fait la promotion depuis des générations; le simulacre, c'est le roman-fleuve, le mouvement prompt de l'écriture qui «divague» des concepts. Pendant cette révolution de l'écriture, les concepts descendent sur la page, déferlent, sans manquer de se transformer, de subir le mouvement intense dela plumequi n'est ni celui dela pageni celui del'écrivant. M ouvement qui est dynamisme pur, hasard de la rencontre. «L e temps approche où il ne sera guère possi ble d'écrire un livre de philosophiecomme on en fait depuissi longtemps» (D R : 4), annonce $D$ eleuze, n'avouant pas que ce désir est le sien et ce livre, I'actualisation même de ce désir. Ainsi, le livre de philosophie, tel l'objet vu, n'est plus désormais un livre de philosophie. Cette dissolution était déjà formulée dans l'exergue, plus haut cité, à propos de l'identité, transformée dans le simulacre, de l'objet lu, en l'occurrence des livres de Joyce et de M allarmé. Ici, D eleuze appliquecette révolution à $D$ ifférence et Répétition.

Pouvons-nous maintenant nous attarder, après la dissolution même de l'objet (livre de philosophie), à celle du sujet. C ar D eleuze, nous l'avons dit, doit, s'il veut renverser la représentation, ébranler égal ement l'identitédu sujet. Aussi conçoit-il ce dernier, non comme une unité interprétative, mais bien comme une multiplicité fêlée. "Cogito pour un moi dissous». «N ouscroyonsà un monde où lesindividuations sont impersonnelles, et les singularités, préindividuelles: la splendeur du "ON "» (DR: 4). N ous retrouvons des indices de cette dissolution identitaire dans $D$ ifférence et Répétition, cette fois appliquée à l'instance narratrice même du texte. Celleci, d'abord, jamais ne se pose comme un JE. Elle est une instance littéralement impersonnelle. Elle se camoufle sous diverses «impersonnalités», qui sont légion dansl'œuvre: Artaud, N ietzsche, Foucault, H egel, Freud, Leibniz, Kant, D escartes, etc. L'instance se disperse, se voile sous ces masques, passe de l'un à l'autre, nomade, fuyante, déguisée. Elleles fait parler; et letexteest le reflet deleur parole. Reflet. O mbre. Simulacre. Le sujet énonciateur est multiple, non divisé ou fractionné, mais en mouvement, dispersé, de l'un à l'autre masque. L'instance donne même la parole au pronom impersonnel par excellence, forme indéterminée et surdéterminée: «V oilà cequ'on nousdit: [...]» (D R : 18). Ce $\mathrm{O}$ est bien le visage grimé de l'énonciateur multiple, il est tous mais personne à la fois, et a en plus le mérite, dit-on, d'exclure la personne qui parle. 
Une autre stratégie narrative évoquant l'effondrement du moi est mise à l'œuvre dans le texte. «Les cycles et les généralités sont le symbole de la généralité. $M$ ais, de toute manière, la générali ité exprime un point de vue d'après lequel un terme peut également être changécontre un autre, un terme, substitué à un autre [...]» (DR: 9). Ce qui est remarquable dans ce passage, c'est le procédé, infime (trois mots!), où se dissout l'instance, presque imperceptiblement. «D u point de vue» de la généralité. L'instance narratrice se cache ici derrière le point de vue d'un pur objet conceptuel. C'est commesi leconcept arrivait vraisemblablement à s'exprimer dans le texte, enveloppé dans l'instance énonciatrice qui lui laisse la place. Le texte subjectivise le concept au profit d'un effacement marqué de l'instance. Stratégie narrative, faut-il le dire, fréquemment utilisée dans I'œuvre, qui participe de la dissolution de l'instance: ce n'est pas elle qui sait, c'est le concept; comme plus tôt, nous le soulevions, c'étaient Artaud, $\mathrm{N}$ ietzsche, etc. qui parlaient, du moinsqui prêtaient leur masque au discours. En apparence (reflet).

$N$ ous avons dit que $D$ ifférence et Répétition se référait à la littérature, puis à l'art pictural afin d'exprimer le simulacre. Le théâtre également est convoqué à ce titre et assure, encore et davantage, l'actual isation d'une pensée sans image et apporte l'essence première, fondamentale, d'une telle agression.

Les signes sont les véritables éléments du thêâtre. IIs témoi gnent des puissancesde la natureet de l'esprit qui agi ssent sous les mots, les gestes, les personnages et les objets représentés. Il s s ignifient la répétition comme mouvement réel, par opposition à la représentation comme faux mouvement de l'abstrait. (D R : 36)

Thêâtre de représentation que $D$ eleuze oppose au théâtre du mouvement qui, lui, nelai sse plus subsister l'identitéd'une chosereprésentée, «thêâtredeproblèmeset dequestionstoujours ouvertes, entraînant le spectateur, la scène et les personnages dans le mouvement réel d'un apprentissage [...]» (D R : 248). $Q$ uel que chose passe entre le sujet et l'objet, ne prenant origineni dansl'un ni dans l'autre. D eleuze rappelleaussi que: «Le thêâtre, c'est le mouvement réel; et de tous les arts qu'il utilise, il extrait le mouvement réel » (DR: 19). D ifférence et Répétition, également, sera au service du théâtre, afin que celui-ci extraie le mouvement réel de l'œuvre.

C ette question à propos des «signes» et del'apprentissage nous amène à considérer un autre aspect de cette pensée. $D$ ans Proust et les signes, D eleuze établissait la théorie selon laquelle il n'y a d'apprentissage que par le corps à corps de deux signes; rencontre sensible, empirique. Dynamisme. Celui qui apprend réellement n'est pas celui qui regarde comment on fait, mais qui «fait avec» celui qui fait, qui participe au mouvement réel du signe; celui qui, pour reprendreun exempledu philosophe, s'agite sur un tabouret et mime le mouvement de la vague, imitant, répétant les gestes du maittrenageur, celui-làn'apprend pas ànager. M ais l'autrequi devient le prolongement corporel dela vagueellemême (son interprétant, dirait une autre doctrine), en mouvement avec elle, qui devient l'autre signe de la vague, en symbiose (en sémiose) dansl'apprentissage, celui-ci apprend réellement. I miter le mouvement de la vague, lui ressembler, à plat ventre sur son tabouret, c'est le lieu de la copie. Participer à son mouvement réel, recevoir «directement»le signe de la vague, dynamisme pur, c'est celui du simulacre. Ainsi, lorsque Deleuze parle du signe comme véritable élément du théâtre, il entend ce mouvement d'apprentissage, d'interaction cruelle entre les corps dans le temps et l'espace de la pièce. Dynamique propre aux signes. II y a quelque chose de résolument peircien dans cette conception du simulacre-signe présenté comme étant action.

\section{Le mouvement de l'écriture (écriture-simulacre)}

$M$ ais comment ce mouvement théâtral peut-il être réal isé dans l'écriture, car c'est bien là la question débattue dans D ifférence et Répétition, et le défi que cette œuvre entend relever? Comment arrive-t-on à faire de son texteun theâtre de la cruauté, qui affirmela dissolution de la pensée, sa fêlure, de même que l'identité effondrée de l'objet? D e quelle manière $D$ eleuze réal ise-t-il le simulacre dans l'écriture?

Le mieux serait de dire, même si la formule est peu heureuse, que le concept descend sur la page. Car il faut, dansle simulacre, que le concept soit mis en scène, projeté sur la page par le mouvement cruel de l'écriture. II faut que le concept prenne vie. Son identité préalable, sa signification ne doivent plus être ce qui importe, elles ne doivent plus primer. Car, dès le passage à l'acte, cette signification, ce fondement seront violés, et ne seront plus les mêmes. IIs se verront transformés par l'action. Submergés. Les meilleures idées ne subsistent pas à l'écriture, désormais soumise à la contrainte de la sémiose. Si la page reçoit le concept tel qu'il est esquissé dansl'esprit, il y a rapport de ressemblance, donc d'identité. Le monde de la représentation est alors sollicité Si le concept, au contraire, ne résiste pas à l'écriture, si cette dernièreletransforme par son élan et son mouvement même, nous vivons le règne du simulacre. Le sujet, dans un tel régime, n'est plus celui qui possède préalablement lemodèle de l'écriture. Sa pensée ne peut pas s'imposer au texte. D ès la projection sur la page, dans le mouvement del'écriture, la 
pensée voit son modèle, celui dont le texte devait être une copie, se transformer. La pensée n'est plus l'élément actif qui «dicte» le texte. Elle reçoit une contrainte extérieure: celle du hasard de la rencontre, celle du mouvement. D eleuze affirme que «[...] tout dynamisme est une catastrophe» (DR : 282). II utilise, pour représenter cette réal ité, I'exemple des plus durs rochers qui, à l'échelle géologique d'un million d'années, passé leur temps d'actualisation, deviennent des matières molles et fluides. $D$ ans la réalité, donc, aucune identité n'est durable et chacune doit être conçue comme un flux. Elleglisse, fuit, derrière une multitude de masques. Ainsi, D eleuze pose le sujet comme larvaire, en cela qu'il se conformeà toutes les positions tortionnaires quel'évolution lui impose.

II n'y a pas de chose qui ne perde son identité telle qu'elle est dans le concept, quand on découvre l'espace et le temps dynamiquesdesa consitution actuelle. [...] C ar nousnesommes pas fixésà un état ou à un moment, mais toujours fixés dans un mouvement en train de se faire. (D R : 282-283)

L'Anti- $\mathbb{E}$ dipe naissait du même désir de briser la repré sentation afin de retrouver ce monde perdu dont elle est la trace, la marque. Le désir, dans ce cas précis, et non plus les «forces» sous la représentation, est l'essence de ce monde perdu.

T outela production désiranteest écrasée, soumiseaux exigences de la représentation, aux mornes jeux du représentant et du représenté dans la représentation. Et c'est là l'essentiel: la reproduction du désir laisseplaceà une simple représentation.

(AO : 63-64)

Le penseur reproche à la représentation de cadavériser les forces, l'intensitéet le désir qui sont à son origine, qui sont au principe de sa genèse. M édiation où tout est figé, fixé, mort. Chaque fois que la production, au lieu d'être saisie dans son originalité, danssa réalité, setrouveainsi rabattuesur un espace de représentation, elle ne peut plus valoir que par sa propre absence, et apparaît comme un manque dans cet espace. [...] Les signes du désir, n'étant pas signifiants, ne le deviennent dans la représentation qu'en fonction d'un signifiant de I'absence ou du manque. (AO : 365 et 369)

Aussi le philosophe entend-il découvrir le mouvement originel, la force, l'intensité, le désir. «[...] la pensée moderne [...] naît de la découverte de toutes les forcesqui agissent sous la représentation del'identique» (D R : 1 )... Car sousle «même» del'I dée, toute une multiplicité gronde.
Ainsi, l'écriture, en respectant le hasard du mouvement, peut atteindre cette force d'origine. Elle est créatrice et doit demeurer mouvement. L'écriture doit se soustraire à toute médiation susceptible de la mettre en boîte, sous vide, immuable, pour toujours; tel que cela se produit dans la repré sentation où l'identité du concept est sédentaire. À propos de Kierkegaard et de $\mathrm{N}$ ietzsche, D eleuze affirme:

Cequi est en question danstouteleur cenvre, c'est lemouvement. [...] Ils veulent mettre la métaphysique en mouvement, en activité. IIsveulent la faire passer à l'acte, et aux mouvements immédiats. II ne leur suffit donc pas de proposer une nouvelle représentation du mouvement; la représentation est déà médiation. II s'agit au contraire de produire dans I'œurve un mouvement capable d'émouvoir l'esprit hors de toute représentation; il s'agit defaire du mouvement lui-même une ceuvre, sansinterposition ; desubstituer dessignesdirectsà des représentations médiates; d'inventer des gravitations, des rotations, des tournoiements, des danses et des sauts qui atteignent directement l'esprit. Cela, c'est une idée d'homme de thêâtre. (DR: 16)

Cette idée d'inventer dans la philosophie un incroyable équival ent du théâtre, peut-on le croire, est égal ement l'espoir de D eleuze. Et cette poétique, qu'il lit dans $\mathrm{N}$ ietzsche et Kierkegaard, révèle le procès d'uneidentification projective: cette poétique, c'est précisément $D$ ifférence et Répétition qui veut l'appliquer, et veut en faire la promotion.

Cequelemonde du simulacre doit permettre, en somme, c'est une pure correspondance entre des parties, une pure interaction d'éléments sans médiation aucune. U ne danse, un saut de l'esprit. U ne pensée sans image. Un véritable apprentissage. «Empirismedu concept». Plusieurs formules de D ifférence et Répétition font insistance à ce sujet:

D ans le thêatre de la répétition, on éprouve des forces pures, des tracés dynamiques dans l'espace qui agi ssent sur l'esprit sans intermédiaire; (p. 19)

La forme qui se réfléchit dans ce fond n'est plus une forme, maisuneligneabstraiteagi ssant directement sur l'âme; (p. 44) [...] le théâtre de la cruauté [...] est un «espace agité», mouvement de gravitation tournant et blessant capable detoucher directement l'organisme. (p. 282)

Communication parfaite qui se produit entre les corps, par leur rencontre, dans un même espace-temps.

La proposition linguistique doit conserver sa fonction de signe et ne pas prétendre trop rapidement à celle de représentation. L'esprit, l'âme et l'organisme réagissent à 
partir d'un signe, d'une force, d'une intensité masquée, et non au contact d'une représentation. Le signe n'est pas médiation, il est la chose, la force et l'intensité même qui éveillent l'esprit, qui forcent à penser. Le signe permet de penser sans image, tel que cela se produit au cours d'un apprentissage. Pour D eleuze le mot est branché sur son origine en mouvement:

Le seul danger, en tout ceci, c'est de confondre levirtuel avec le possible. Car le possiblesoppose au réd [...] On aurait tort de nevoir ici qu'une dispute demots: il s'agit del'existence ellemême. ( D R : 272-273)

\section{D ifférence et Répétition comme signe}

Ainsi, D ifférence et Répétition devient réellement un simulacre, lorsqu'il entraîne son lecteur dans son mouvement spiral, dans son tourbillon. II devient un signe qui rejoint le lecteur sansmédiation aucune, qui leforce et l'obligeà participer à sa folie, à son mouvement. II force le lecteur à penser, perdu qu'est ce dernier à chercher le bon chemin de l'interprétation et surtout le bon point de départ de sa promenade lectorale. De plus, à bien lire, on remarque que l'ouvrage se pose littéralement comme un signe à décoder. Le lecteur est en apprentissage. «Car nousne savonspasencore» (D R : 40); «[...] nousnesommespasencoreen état de comprendre[...] »(PS: 18 et 46). Le lecteur est «[...] entraîné [...] dans le mouvement réel d'un apprentissage» (D R : 248). Et lorsqueD eleuzedit, à propos du simulacre, qu'il est construit sur unedisparité, qu'il a intériorisé la dissimilitude de ses sériesconstituantes et qu'il raconte plusieurs histoi resà la fois, on est surpris de constater l'étrange application decettephraseautant àcequ'il nommesimulacrequ'àl'œuvre qu'il a élaborée. U ne phrase de L ogi quedu senspose clairement le roman - là où on raconte des histoires - comme un simulacre: «0 n sait par exemple que certains procédés littéraires permettent de raconter plusieurs histoires à la fois» (LS: 300). Différence et Répétition est bien le lieu où se racontent des histoires, celle de l'art, du théâtre, des mathématiques, de la biologie, de la logique, de la philosophie, de l'apprentissage, du simulacre, etc. Labouclesereferme: I'ouvrage philosophique queD eleuze compareau genrelittéraire, cet ouvrage qui aspire explicitement à l'œuvre d'art et à la science-fiction, vise véritablement l'actualisation du simulacre, multipliant les séries pour occasionner la «multi-lecturepossible» de l'œuvre.

$C^{\prime}$ 'est également en tant que signe que le texte devient un labyrinthe où le lecteur doit, dans la symbiose de l'apprentissage, décoder et décrypter cessignes. M ouvement dela vague qui submergelelecteur, qui l'entraîne. M aispour cela, il devient nécessaire desupposer l'existenced'un lecteur idéal : celui qui a le désir de commencer au bon endroit, et le désir éperdu de trouver cet endroit. Le lecteur ainsi est pris comme le modèle, selon son identité fixe et immuable d'interprétant. Renverser le modèle ne consiste pas à conclure à une définition contraire à celui-ci, à un concept dont l'identité est opposée à celle du modèle. Renverser le modèle, afin d'aboutir au simulacre, consiste plutôt à déjouer le lecteur: quand ce dernier attend I'indication d'un commencement, il s'agit de noyer ce commencement dans l'œuvre et defaire en sortequelelecteur en poursuivetout demêmela quête, mal gréson naufrage. Q u'il apprenne enfin qu'on ne commence pas et qu'on ne le peut pas réellement. Lesimulacre, c'est lemouvement decette dérive, c'est l'expérience de cette perdition.

Ainsi, D eleuze finit-il par échapper à la dialectique, au simple rapport logique de renversement, en définissant le simulacre, non plus comme le contraire du modèle, mais, à un autre niveau, comme l'apprentissage même de celui-ci. On dénie le modèle, mais cet exercice ne nous mène pas à une autre position logique, à une autre représentation qui s'opposeà la première et la suppose. Lepenseur nous propose le tournoiement du simulacre et l'agitation du rêve. II nous proposel'apprentissage d'un signe, au profit deson immuable compréhension. II nousproposeD ifférenceet Répétition, dont la lecture est d'autant plus intense, et tient par trop le lecteur en éveil, en dérive, qu'on cherche toujours, et non sans difficultés, à traduire ce discours, pas tant hermétique que scientifique et artistique à la fois. O n gravite autour de l'œuvre, autour de ses précieuses et belles formulations, non sans ressentir «l'effet de littérature» - que j'ai présenté au départ comme un effet delabyrinthe - si naturel à D ifférence et Répétition.

O n referme le livre. On se réveille. O n a rêvé au labyrinthe. O n ouvrelesyeux et notre chambreest toujours notre chambre. Et le couloir mène toujours au salon. D ans notre rêve, tout avait changé. J usqu'à nous. Q ui rêvait réellement? Y a-t-il eu rêve? A-t-il fini?

\section{RÉFÉRENCES BIBLIOGRAPHIQUES}

D eleuze, G. [1969]: D ifférence et Répétition (D R), Paris, P.U .F., 408p. ; [1964] : Proust et lessignes (PS), Paris, P.U.F., 195 p.;

[1972]: L'Anti-〔E dipe, Capitalisme et Schizophrénie (AO ), Paris, Éd. de M inuit, coll. «Critique», $470 \mathrm{p}$. ;

[1969] : Logiquedu sens(LS), Paris, Éd. deM inuit, coll. «C ritique», 392 p. 